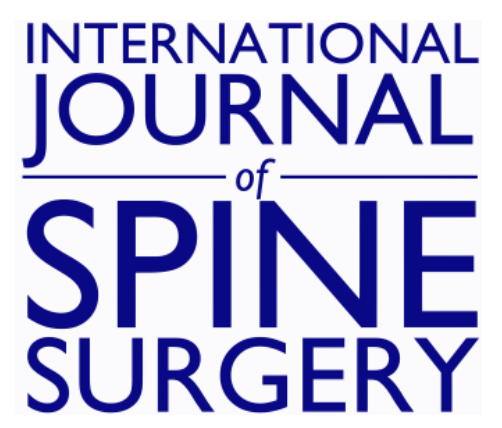

\title{
Enhancing Evaluation of Cervical Spine: Thresholds for Normal CT Relationships in the Subaxial Cervical Spine
}

CATHERINE W. CAHILL, KRISTEN E. RADCLIFF and CHARLES A. REITMAN

Int J Spine Surg 2018, 12 (4) 510-519

doi: https://doi.org/10.14444/5062

http://ijssurgery.com/content/12/4/510

This information is current as of April 25, 2023.

Email Alerts Receive free email-alerts when new articles cite this article. Sign up at:

http://ijssurgery.com/alerts

The International Journal of Spine Surgery

2397 Waterbury Circle, Suite 1,

Aurora, IL 60504, Phone: +1-630-375-1432 


\title{
Enhancing Evaluation of Cervical Spine: Thresholds for Normal CT Relationships in the Subaxial Cervical Spine
}

\author{
CATHERINE W. CAHILL, MD, ${ }^{1}$ KRISTEN E. RADCLIFF, MD ${ }^{2}$ CHARLES A. REITMAN, MD $^{3}$ \\ ${ }^{I}$ Department of Orthopedic Surgery, Baylor College of Medicine, Houston, Texas, ${ }^{2}$ Thomas Jefferson University Department of Orthopedic Surgery, Egg Harbor, \\ New Jersey, ${ }^{3}$ MUSC Department of Orthopaedics, Charleston, South Carolina
}

\begin{abstract}
Background: Very little normative computed tomography (CT) scan data exist defining expected relationships of vertebral structures in the intact cervical spine. Better understanding of normal relationships should improve sensitivity of injury detection, particularly for facet subluxation. The purpose of this paper was to describe the normal anatomical relationships and most sensitive measurements to detect abnormal alignment in the subaxial cervical spine.

Methods: A group of $30 \mathrm{CT}$ scans with no documented cervical spine injury were utilized from an established database in a trauma population. Twenty-two anatomical measurements were made for each level of the subaxial cervical spine using Microview software. For the purposes of measurement, the upper confidence limit of normal was reported as two standard deviations from the mean.

Results: The novel, CT based measurements of bone articulation were generally smaller and had lower confidence intervals compared to traditional radiographic measurements of midline structures (such as interspinous distance, interlaminar widening, disc space widening). The upper limit of normal of facet joint height was reported (1.54 mm anterior, $1.27 \mathrm{~mm}$ posterior, and $2.0 \mathrm{~mm}$ midportion), which may help identify distractive-flexion injuries. The upper limit of normal vertebral translation $(2.0 \mathrm{~mm})$ was also reported to identify translation/rotation injuries.

Conclusions: Normal CT measurements for the subaxial cervical spine, especially in the facets, were found to have small confidence limits and variation. Based upon these findings, we conclude that facet measurements and translation may be better screening tools than traditional radiographic criteria based upon midline structures. Using these measurements may improve detection of cervical spine injuries warranting further imaging or investigation and reducing missed injuries.

Clinical Relevance: Improved understanding of normal anatomic measures in the subaxial spine will allow for better screening and identification of injuries.

Cervical Spine

Keywords: cervical spine, intervertebral measurements, CT scan
\end{abstract}

\section{INTRODUCTION}

Historically, traumatic cervical spine injuries were identified using radiographs. Assessment of radiographs was performed using defined radiographic landmarks such as the spinolaminar line, and bony relationships, such as the interspinous distance (ISD) or atlanto-dental interval. However, recently, high resolution computed tomography (CT) scan has become ubiquitous at most trauma centers. CT scan has slowly supplanted radiographs as the trauma screening modality of choice. In 2009, guidelines were published based on literature review advocating for CT scan as the primary radiologic screening modality in trauma patients. ${ }^{1}$ The basis for this recommendation was the superior sensitivity of CT $(90 \%-100 \%)$ for detecting traumatic bony injury, making plain radiographs in this setting almost obsolete. $^{2-7}$

Although CT is commonly used to identify fractures and to screen trauma patients, there is very little published data regarding normal subaxial spine relationships on $\mathrm{CT}$. Normal bony relationships, such as ISD, have not been well defined despite the superior resolution and lower susceptibility to magnification error. The purpose of this study was to describe normal anatomical features, including CT based landmarks, of the subaxial disks and facet joints of the cervical spine on CT scan in an asymptomatic population. This information may lead to the creation of sensitive screening parameters that could enable identification of subtle articular displacement and posterior ligamentous injury. Better understanding of normal anatomy 


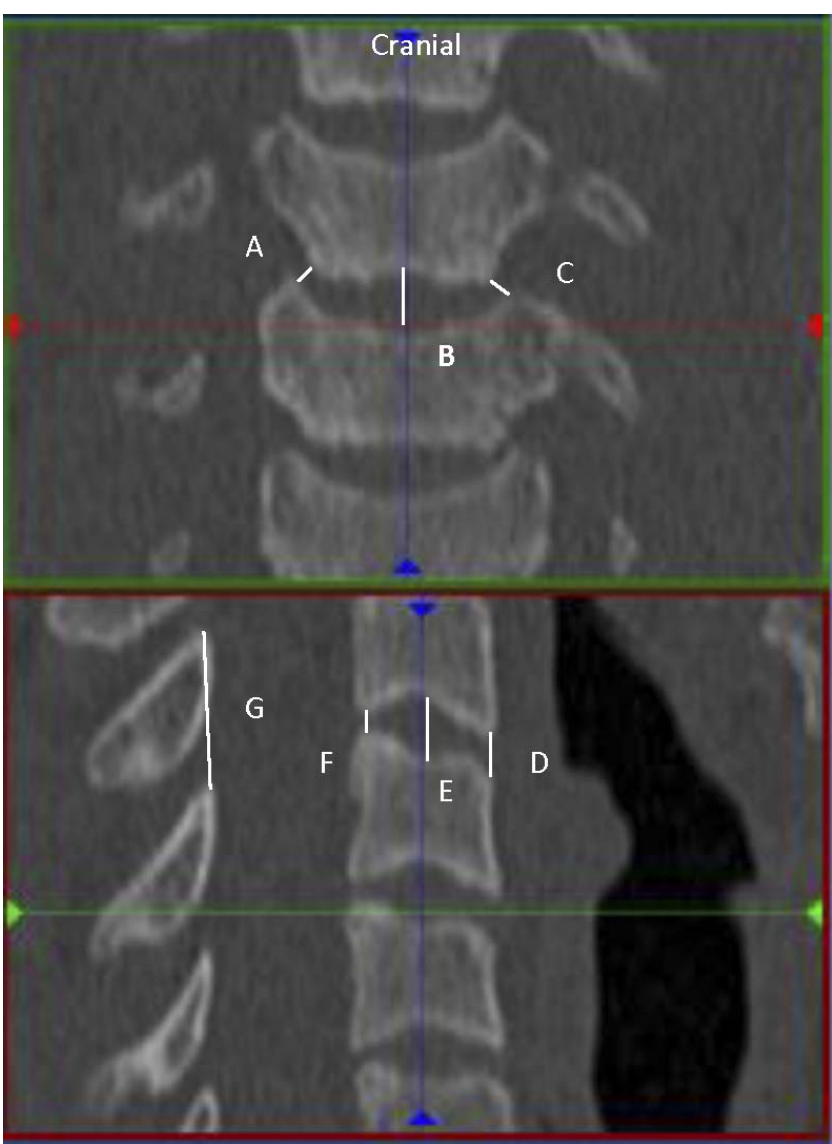

Figure 1. Coronal and sagittal disc measurements. Right UVJ (A), coronal mid disc (B), left UVJ (C). Anterior DH (D), sagittal mid disc (E), posterior DH (F), ISD (G).

should improve our ability to detect injury, particularly in those patients with more subtle characteristics of damage. ${ }^{8}$

\section{MATERIALS AND METHODS}

Permission for evaluation of patient data was obtained through the Institutional Review Board. A database of 100 screening cervical spine CT scans was available, which had been collected for the purpose of multiple evaluations. This database was a random sample of studies collected on patients who presented to the emergency room of a level I trauma center and underwent screening CT. ${ }^{9}$ All of these exams were determined to be free of acute cervical spine injury by the faculty radiologist, and had no clinical evidence of injury at discharge or short-term follow-up. For this study, the first 38 scans were evaluated. Eight patients were excluded. Reasons for exclusion included facial fracture, skull fracture, neurologic injury without evidence of cervical injury, and congenital abnormalities. Due to the precision of CT scan and the absence of information about the variability of measurement in the population, a sample size calculation was performed after a pilot analysis was performed. After identification of the standard deviation (SD) and mean measurements, a power calculation was performed after $10 \mathrm{CT}$ scans were measured, showing that $30 \mathrm{CT}$ scans would identify a $95 \%$ confidence interval (CI) for the coronal and sagittal facet measurements. Final power analysis confirmed an adequate sample size at the conclusion of the analysis.

All measurements were made using Microview 3D Image Viewer and Analysis Tool (Parallax innovations, http://microview.sourceforge.net/) software. Microview is an open source, advanced image viewer software. This software measures to the 100th millimeter. Measurements were rounded to the nearest 10th of a millimeter and recorded. All images were reoriented using the software to take the most accurate measurement. Thus, the images were reoriented so that measurements were precisely perpendicular to the plane of the anatomy. Microview allows reformatting of coronal, axial, and sagittal planes making this possible for every level and every joint. After the images were appropriately oriented, the actual distance was taken from cortical edge to cortical edge at each desired joint, disc, or ratio. Images were magnified to facilitate accurate measurements. Cortical edge was chosen to be the point midway between whitest white and darkest dark on the images. This allowed for consistency between measurements. All measurements were done by the primary author.

Twenty-two measurements were made for each level of the subaxial cervical spine from $\mathrm{C} 2-3$ to C67. Nine coronal measurements were obtained including left and right uncovertebral joints (UVJs), left and right medial, lateral and mid facets, and mid disc height (DH). The 11 sagittal measurements obtained included ISD, translation, left and right anterior, posterior and middle facet, and anterior, posterior, and mid DHs. Two additional ratios were obtained on the sagittal cuts to measure the amount of facet translation. This measurement was the ratio of covered to uncovered facet for the right and left facets at each level. Figures 1 and 2 show how individual measurements were obtained.

For the coronal images, UVJ measurements were obtained at the inferolateral corner of the vertebral body perpendicular to the joint (Figure 1, A and C). 

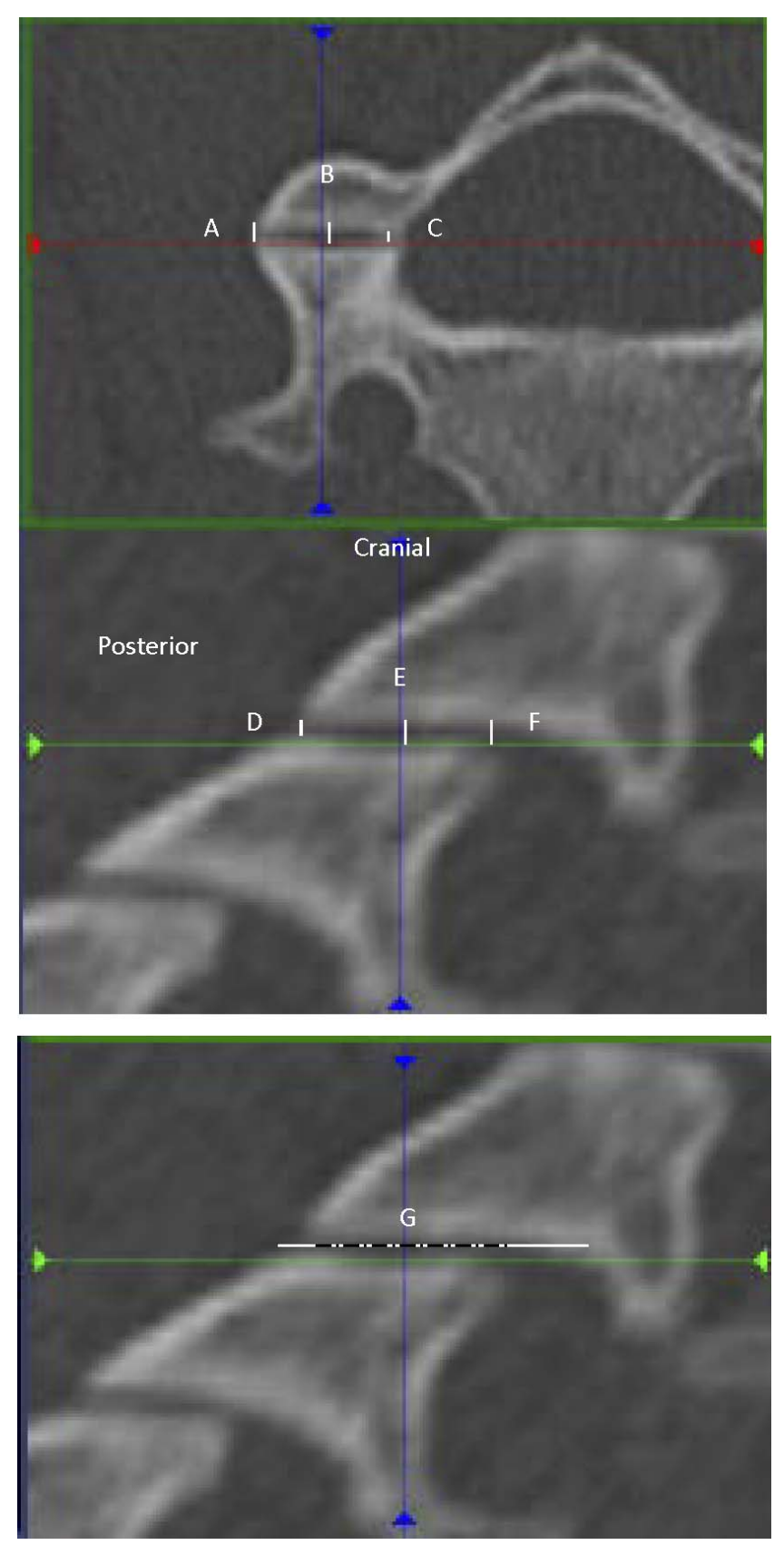

Figure 2. Axial and sagittal facet measurements. Lateral facet (A), axial mid facet $(B)$, medial facet $(C)$. Posterior facet $(D)$, sagittal mid facet $(E)$, anterior facet $(F)$. Facet overlap $(G)$.

DH measurement was the height of the disc midway between the two UVJ measurements. In the coronal plane, three facet joint space measurements were obtained: lateral, midpoint, and medial (Figure 2, A, B, and C). Mid facet joint space was obtained midway between the lateral and medial facet measurements, which were measured at the most medial and most lateral edge of the joint perpendicular to the bone edges.
Posterior, midpoint, and anterior facet joint space measurements were obtained from the sagittal reconstructions (Figure 2, E, E, and F) using the most anterior and posterior edges of the facet and the point midway between the two for the middle facet measurement. Percent of facet overlap was also obtained on the sagittal view as the amount of superior and inferior facet, which covered each other to the entire length from the most posterior aspect of the caudal facet to the most anterior aspect of the cranial facet (Figure 2G). Sagittal DH was obtained at the most anterior, midpoint, and posterior edges of the vertebral body, always perpendicular to the cortical edges (Figure 1, D, $\mathrm{E}$, and F). Translation was measured at the anterior border of the vertebral bodies. A line parallel to the anterior cortical edge of the cranial and caudal vertebrae was drawn, and the distance between these lines represented the anterior/posterior translation of the bodies to one another. The final sagittal measurement, ISD, was taken between the most posterior/superior corners of the spinous processes (Figure 1G).

All measurements were recorded in an excel spreadsheet. SPSS (IBM Analytics) for excel (Microsoft) software was used for statistical analysis. Population CIs (as opposed to CIs of the mean) were calculated for each measurement both for individual levels and as a group by identifying the values that were two SDs from the mean. A 95\% CI was chosen so that there would be greater sensitivity to detect abnormal measurements. Left and right side measurements were compared and combined as there was no statistically significant difference between sides. This in effect doubled the sample size. In addition, analysis of variance between levels was done for each measurement.

\section{RESULTS}

The mean age was 39 (SD 15). The population was $65 \%$ male. There were seven patients over the age of 65 . Lower and upper $95 \%$ confidence limits for the measurements are shown in Table 1. For all facet measurements the upper limit of the CIs was small. As expected, the upper limit of normal was also consistent between sides $(P<.05)$. Thus right and left side data were pooled for further analysis (Figure 3; Table 2). At all levels, the mean anterior (mean 0.87, SD 0.03), posterior (mean 0.68, SD 0.02 ), medial (mean 0.58, SD 0.02), or lateral border (mean 0.51, SD 0.02) facet height was less than 1 
mm. At all levels, the upper 95\% CI for anterior $(1.54 \mathrm{~mm})$, posterior $(1.27 \mathrm{~mm})$, medial $(1.11 \mathrm{~mm})$, or lateral $(1.01 \mathrm{~mm})$ facet height was less than $2 \mathrm{~mm}$. Of the levels, C56 appeared to have the largest 95\% upper CI (1.69 anterior, 1.35 posterior, 1.32 medial, 1.05 lateral). The midfacet measurements appeared to have higher variation than the anterior, posterior, medial, and lateral measurements (midpoint facet mean 1.14, SD 0.04, upper 95\% CI 2.09) (Table 2).

The upper $95 \%$ CI of uncovertebral average distance for all levels was $3.08 \mathrm{~mm}$ (Table 2; mean 1.6, SD 0.06, greatest value $3.4 \mathrm{~mm}$ at $\mathrm{C} 23$ ). However, there were significant differences between levels with C56 and C67 having the lowest upper CI limit (approximately 0.9; Table 2).

None of the remaining measurement had statistical differences between levels including interspinous and disc space distances. Relative to facet joint distances, there was wider variation and CIs were greater. At the disc midpoint the upper limit for both the sagittal measurements did exceed $5 \mathrm{~mm}$ (mean 4.9, SD 0.09, 95\% CI $6.9 \mathrm{~mm}$ ). For ISD the upper CI limit was 18.6 (mean 14.3, SD 0.18) and for translation the upper limit was $2.04 \mathrm{~mm}$ (mean 0.39 , SD 0.07).

\section{DISCUSSION}

These results provide valuable reference data for interpreting CT scans of the subaxial spine. Overall, we found that bony landmarks similar to those traditionally used on radiographs could be reproduced on CT scan along with novel measurements that were previously impossible, such as UVJ height. The novel measurements of bone articulation were generally smaller and had lower CIs compared to traditional radiographic measurements of midline structures (such as ISD, interlaminar widening, disc space widening). We defined the upper limit of normal of facet joint height $(1.54 \mathrm{~mm}$ anterior, $1.27 \mathrm{~mm}$ posterior, and $2.0 \mathrm{~mm}$ midportion) to identify distractive-flexion injuries. We also defined normal vertebral translation $(2.0 \mathrm{~mm})$ along a posterior vertebral body line in the true sagittal plane to identify translation/rotation injuries. Based upon these findings, we conclude that facet measurements and translation may be better screening tools than traditional radiographic criteria based upon midline structures.

Previous screening criteria for injury in the subaxial spine are based on radiographic alignment of midline structures. ${ }^{10}$ Concepts such as the spinolaminar line and posterior vertebral body line $^{11}$ were created to represent relationships between bones because it was impossible to precisely measure sub millimeter measurements on radiographs and compare between centers. Although these concepts were widely used, they are susceptible to magnification error and projection error. Additionally, the modality upon which they are commonly based (conventional radiography) is no longer widely used in trauma screening. With conventional radiography, it was not possible to precisely, reliably measure subtle relationships of articulating bones (such as facet joints or UVJs). The purpose of this study was to explore normal CT scans to identify bony relationships that may be useful as CT based injury screening parameters.

Currently, CT scan is used for identification of bony pathology and magnetic resonance imaging (MRI) is performed to identify discoligamentous complex injury. CT scan enables precise measurement of small distances in narrow anatomical regions. ${ }^{9}$ The purpose of this investigation was to report the precise anatomical bony relationships of articulating structures in the subaxial spine based on CT scan. We hypothesize that these will be useful in identification of occult ligamentous complex injury in the cervical spine in the setting of trauma and therefore expedite patient care, although that will have to be subject to further clinical study.

The most common occult ligamentous injury in the absence of fracture in the subaxial spine is isolated posterior ligamentous complex injury and/ or injury to the facet complex. Our measurements indicate that the articular facet height is highly conserved in the subaxial spine. The mid-facet height in the subaxial spine should be less than 2 $\mathrm{mm}$. The anterior and posterior facet height should be less than $1.5 \mathrm{~mm}$. Although facet joint distraction and diastasis is considered to be a sign of an AllenFerguson distraction-flexion stage 2 injury, the normal facet joint height based upon CT scan has not been reported to our knowledge. Previous technology based on radiographs was too insensitive to identify these small measurements. Currently MRI is performed on patients to screen for occult distractive flexion injuries even in the presence of a grossly normal CT scan. However, MRI is expensive, time consuming, and technically cumbersome in unstable trauma patients. We expect that future studies may determine whether utilization of these 
Table 1. Descriptive statistics of cervical measurements.

\begin{tabular}{|c|c|c|c|c|c|c|c|c|c|c|}
\hline & \multirow[b]{2}{*}{$N$} & \multirow[b]{2}{*}{ Mean } & \multirow[b]{2}{*}{ SD } & \multirow{2}{*}{$\begin{array}{c}\text { Standard } \\
\text { Error }\end{array}$} & \multicolumn{2}{|c|}{ 95\% CI for Mean } & \multirow[b]{2}{*}{ Minimum } & \multirow[b]{2}{*}{ Maximum } & \multirow[b]{2}{*}{ 95\% Upper CI } & \\
\hline & & & & & Lower Bound & Upper Bound & & & & 95\% Lower CI \\
\hline Left UVJ ( & ronal & & & & & & & & & \\
\hline $\mathrm{C} 23$ & 58 & 1.79 & .853 & .158 & 1.47 & 2.12 & 1 & 4 & 3.50 & 0.09 \\
\hline $\mathrm{C} 34$ & 58 & 1.83 & .756 & .140 & 1.54 & 2.12 & 1 & 4 & 3.34 & 0.32 \\
\hline $\mathrm{C} 45$ & 58 & 1.44 & .586 & .111 & 1.22 & 1.67 & 0 & 3 & 2.62 & 0.27 \\
\hline C56 & 58 & 1.33 & .524 & .097 & 1.13 & 1.53 & 0 & 2 & 2.37 & 0.28 \\
\hline C67 & 58 & 1.93 & .599 & .111 & 1.70 & 2.16 & 1 & 3 & 3.13 & 0.73 \\
\hline Average & 290 & 1.67 & .706 & .059 & 1.55 & 1.78 & 0 & 4 & 3.08 & 0.25 \\
\hline Right UVJ & corona & & & & & & & & & \\
\hline $\mathrm{C} 23$ & 29 & 1.6990 & .80166 & .14886 & 1.3940 & 2.0039 & .68 & 4.10 & 3.30 & 0.10 \\
\hline C34 & 29 & 1.5914 & .83313 & .15471 & 1.2745 & 1.9083 & .52 & 4.12 & 3.26 & 0.00 \\
\hline $\mathrm{C} 45$ & 29 & 1.3724 & .63461 & .11784 & 1.1310 & 1.6138 & .00 & 3.41 & 2.64 & 0.10 \\
\hline C56 & 29 & 1.2145 & .60589 & .11251 & .9840 & 1.4450 & .00 & 2.51 & 2.43 & 0.00 \\
\hline C67 & 29 & 1.8093 & .82818 & .15379 & 1.4943 & 2.1243 & .81 & 3.57 & 3.47 & 0.15 \\
\hline Average & 145 & 1.5373 & .76824 & .06380 & 1.4112 & 1.6634 & .00 & 4.12 & 3.07 & 0.00 \\
\hline Middle disc & $(\operatorname{mid} \mathrm{c}$ & ronal) & & & & & & & & \\
\hline C23 & 29 & 4.9972 & .73483 & .13645 & 4.7177 & 5.2768 & 3.25 & 6.19 & 6.47 & 3.53 \\
\hline C34 & 29 & 4.7810 & 1.04291 & .19366 & 4.3843 & 5.1777 & 2.22 & 7.10 & 6.87 & 2.70 \\
\hline $\mathrm{C} 45$ & 29 & 4.8176 & 1.15149 & .21383 & 4.3796 & 5.2556 & 1.12 & 6.48 & 7.12 & 2.51 \\
\hline C56 & 29 & 4.6193 & 1.09779 & .20386 & 4.2017 & 5.0369 & 1.93 & 6.62 & 6.81 & 2.42 \\
\hline C67 & 29 & 5.0262 & .95944 & .17816 & 4.6613 & 5.3912 & 2.52 & 6.95 & 6.95 & 3.11 \\
\hline Average & 145 & 4.8483 & 1.00505 & .08347 & 4.6833 & 5.0133 & 1.12 & 7.10 & 6.86 & 2.84 \\
\hline Left medial & acet ( & oronal) & & & & & & & & \\
\hline C23 & 29 & .5759 & .24939 & .04631 & .4810 & .6707 & .13 & 1.08 & 1.07 & 0.08 \\
\hline C34 & 29 & .5945 & .23343 & .04335 & .5057 & .6833 & .17 & 1.00 & 1.06 & 0.13 \\
\hline $\mathrm{C} 45$ & 29 & .5117 & .27290 & .05068 & .4079 & .6155 & .17 & 1.21 & 1.06 & 0.00 \\
\hline C56 & 29 & .7093 & .32663 & .06065 & .5851 & .8336 & .14 & 1.54 & 1.36 & 0.06 \\
\hline C67 & 29 & .4855 & .23009 & .04273 & .3980 & .5730 & .10 & .88 & 0.95 & 0.03 \\
\hline Average & 145 & .5754 & .27265 & .02264 & .5306 & .6201 & .10 & 1.54 & 1.12 & 0.03 \\
\hline Right medi & facet & coronal) & & & & & & & & \\
\hline $\mathrm{C} 23$ & 29 & .6003 & .22422 & .04164 & .5151 & .6856 & .17 & 1.03 & 1.05 & 0.15 \\
\hline C34 & 29 & .6121 & .21333 & .03961 & .5309 & .6932 & .19 & 1.04 & 1.04 & 0.19 \\
\hline $\mathrm{C} 45$ & 29 & .5759 & .25569 & .04748 & .4786 & .6731 & .16 & 1.16 & 1.09 & 0.06 \\
\hline C56 & 29 & .6586 & .31088 & .05773 & .5404 & .7769 & .00 & 1.20 & 1.28 & 0.04 \\
\hline C67 & 29 & .5131 & .22372 & .04154 & .4280 & .5982 & .18 & .96 & 0.96 & 0.07 \\
\hline Average & 145 & .5920 & .24931 & .02070 & .5511 & .6329 & .00 & 1.20 & 1.09 & 0.09 \\
\hline Left midpo & t face & (coronal) & & & & & & & & \\
\hline $\mathrm{C} 23$ & 29 & 1.05 & .406 & .075 & .89 & 1.20 & 0 & 2 & 1.86 & 0.24 \\
\hline C34 & 29 & 1.31 & .465 & .086 & 1.13 & 1.49 & 0 & 2 & 2.24 & 0.38 \\
\hline $\mathrm{C} 45$ & 29 & 1.38 & .416 & .077 & 1.22 & 1.54 & 1 & 2 & 2.21 & 0.55 \\
\hline C56 & 29 & 1.19 & .352 & .065 & 1.05 & 1.32 & 1 & 2 & 1.89 & 0.48 \\
\hline C67 & 29 & 1.02 & .334 & .062 & .89 & 1.15 & 0 & 2 & 1.69 & 0.35 \\
\hline Average & 145 & 1.19 & .417 & .035 & 1.12 & 1.26 & 0 & 2 & 2.02 & 0.36 \\
\hline Right midp & int fac & t (coronal & & & & & & & & \\
\hline $\mathrm{C} 23$ & 29 & 1.0476 & .39446 & .07325 & .8975 & 1.1976 & .58 & 1.86 & 1.84 & 0.26 \\
\hline C34 & 29 & 1.3548 & .43048 & .07994 & 1.1911 & 1.5186 & .54 & 2.49 & 2.22 & 0.49 \\
\hline $\mathrm{C} 45$ & 29 & 1.3528 & .43262 & .08033 & 1.1882 & 1.5173 & .52 & 2.47 & 2.22 & 0.49 \\
\hline C56 & 29 & 1.3369 & .53409 & .09918 & 1.1337 & 1.5401 & .36 & 2.59 & 2.41 & 0.27 \\
\hline C67 & 29 & 1.0597 & .43697 & .08114 & .8934 & 1.2259 & .36 & 2.32 & 1.93 & 0.19 \\
\hline Average & 145 & 1.2303 & .46507 & .03862 & 1.1540 & 1.3067 & .36 & 2.59 & 2.16 & 0.30 \\
\hline Left lateral & acet $(c$ & ronal) & & & & & & & & \\
\hline $\mathrm{C} 23$ & 29 & .5434 & .24766 & .04599 & .4492 & .6377 & .00 & 1.10 & 1.04 & 0.05 \\
\hline C34 & 29 & .4966 & .22241 & .04130 & .4120 & .5812 & .12 & .99 & 0.94 & 0.05 \\
\hline $\mathrm{C} 45$ & 29 & .5366 & .24415 & .04534 & .4437 & .6294 & .12 & 1.11 & 1.02 & 0.05 \\
\hline C56 & 29 & .4907 & .27985 & .05197 & .3842 & .5971 & .00 & 1.28 & 1.05 & 0.00 \\
\hline C67 & 29 & .4717 & .26301 & .04884 & .3717 & .5718 & .00 & .91 & 1.00 & 0.00 \\
\hline Average & 145 & .5078 & .25017 & .02078 & .4667 & .5489 & .00 & 1.28 & 1.01 & 0.01 \\
\hline Right later & facet & coronal) & & & & & & & & \\
\hline $\mathrm{C} 23$ & 29 & .4555 & .22389 & .04157 & .3704 & .5407 & .00 & .98 & 0.90 & 0.01 \\
\hline C34 & 29 & .4648 & .22808 & .04235 & .3781 & .5516 & .17 & .84 & 0.92 & 0.01 \\
\hline $\mathrm{C} 45$ & 29 & .5510 & .25890 & .04808 & .4526 & .6495 & .16 & 1.12 & 1.07 & 0.03 \\
\hline C56 & 29 & .5452 & .29488 & .05476 & .4330 & .6573 & .00 & 1.41 & 1.13 & 0.00 \\
\hline C67 & 29 & .5600 & .20920 & .03885 & .4804 & .6396 & .21 & 1.07 & 0.98 & 0.14 \\
\hline Average & 145 & .5153 & .24573 & .02041 & .4750 & .5556 & .00 & 1.41 & 1.01 & 0.02 \\
\hline ISD (sagitt & & & & & & & & & & \\
\hline $\mathrm{C} 23$ & 29 & 15.7624 & 2.75350 & .51131 & 14.7150 & 16.8098 & 7.70 & 20.95 & 21.27 & 10.26 \\
\hline C34 & 29 & 14.6317 & 1.76001 & .32683 & 13.9623 & 15.3012 & 12.24 & 19.16 & 18.15 & 11.11 \\
\hline $\mathrm{C} 45$ & 29 & 14.0155 & 1.84557 & .34271 & 13.3135 & 14.7175 & 10.09 & 16.52 & 17.71 & 10.32 \\
\hline C56 & 29 & 13.1521 & 1.56348 & .29033 & 12.5574 & 13.7468 & 10.32 & 16.47 & 16.28 & 10.03 \\
\hline C67 & 29 & 14.1241 & 1.81533 & .33710 & 13.4336 & 14.8147 & 11.46 & 19.20 & 17.75 & 10.49 \\
\hline Average & 145 & 14.3372 & 2.14344 & .17800 & 13.9853 & 14.6890 & 7.70 & 20.95 & 18.62 & 10.05 \\
\hline
\end{tabular}




\begin{tabular}{|c|c|c|c|c|c|c|c|c|c|c|}
\hline & \multirow[b]{2}{*}{$N$} & \multirow[b]{2}{*}{ Mean } & \multirow[b]{2}{*}{ SD } & \multirow{2}{*}{$\begin{array}{c}\text { Standard } \\
\text { Error }\end{array}$} & \multicolumn{2}{|c|}{$95 \%$ CI for Mean } & \multirow[b]{2}{*}{ Minimum } & \multirow[b]{2}{*}{ Maximum } & \multirow[b]{2}{*}{ 95\% Upper CI } & \\
\hline & & & & & Lower Bound & Upper Bound & & & & 95\% Lower CI \\
\hline Vertebral b & dy tra & slation $(\mathrm{s}$ & gittal) & & & & & & & \\
\hline C23 & 29 & .5841 & .86261 & .16018 & .2560 & .9123 & -1.09 & 3.86 & 2.31 & 0.00 \\
\hline $\mathrm{C} 34$ & 29 & .5369 & .64093 & .11902 & .2931 & .7807 & -.84 & 1.74 & 1.82 & 0.00 \\
\hline C45 & 29 & .5210 & .72608 & .13483 & .2448 & .7972 & -1.57 & 1.70 & 1.97 & 0.00 \\
\hline C56 & 29 & .0855 & .88789 & .16488 & -.2522 & .4233 & -1.53 & 1.93 & 1.86 & 0.00 \\
\hline C67 & 29 & .2131 & .91067 & .16911 & -.1333 & .5595 & -1.50 & 1.45 & 2.03 & 0.00 \\
\hline Average & 145 & .3881 & .82582 & .06858 & .2526 & .5237 & -1.57 & 3.86 & 2.04 & 0.00 \\
\hline Anterior D & (sagi & & & & & & & & & \\
\hline C23 & 29 & 2.6990 & .83906 & .15581 & 2.3798 & 3.0181 & 1.13 & 4.40 & 4.38 & 1.02 \\
\hline C34 & 29 & 2.8310 & .85043 & .15792 & 2.5075 & 3.1545 & 1.05 & 4.32 & 4.53 & 1.13 \\
\hline $\mathrm{C} 45$ & 29 & 3.4552 & .94400 & .17530 & 3.0961 & 3.8143 & 1.42 & 5.89 & 5.34 & 1.57 \\
\hline C56 & 29 & 3.1310 & 1.15688 & .21483 & 2.6910 & 3.5711 & .00 & 5.19 & 5.44 & 0.82 \\
\hline C67 & 29 & 3.4262 & 1.10487 & .20517 & 3.0059 & 3.8465 & .99 & 5.26 & 5.64 & 1.22 \\
\hline Average & 145 & 3.1085 & 1.02094 & .08478 & 2.9409 & 3.2761 & .00 & 5.89 & 5.15 & 1.07 \\
\hline Middle DH & (sagitt & & & & & & & & & \\
\hline C23 & 29 & 4.888 & .7602 & .1412 & 4.598 & 5.177 & 3.0 & 6.4 & 6.41 & 3.37 \\
\hline C34 & 29 & 4.848 & 1.1929 & .2215 & 4.394 & 5.301 & 1.8 & 7.1 & 7.23 & 2.46 \\
\hline C45 & 29 & 4.922 & 1.1833 & .2197 & 4.472 & 5.372 & 1.1 & 6.7 & 7.29 & 2.56 \\
\hline C56 & 29 & 4.656 & 1.2052 & .2238 & 4.198 & 5.115 & 1.8 & 6.5 & 7.07 & 2.25 \\
\hline C67 & 29 & 5.244 & 1.1833 & .2197 & 4.794 & 5.694 & 1.3 & 6.7 & 7.61 & 2.88 \\
\hline Average & 145 & 4.911 & 1.1191 & .0929 & 4.728 & 5.095 & 1.1 & 7.1 & 7.15 & 2.67 \\
\hline Posterior D & I (sag & tal) & & & & & & & & \\
\hline C23 & 29 & 2.3107 & .70818 & .13151 & 2.0413 & 2.5801 & .99 & 4.45 & 3.73 & 0.89 \\
\hline C34 & 29 & 2.1069 & .74378 & .13812 & 1.8240 & 2.3898 & .43 & 3.56 & 3.59 & 0.62 \\
\hline $\mathrm{C} 45$ & 29 & 2.4521 & .65815 & .12222 & 2.2017 & 2.7024 & 1.07 & 3.74 & 3.77 & 1.14 \\
\hline C56 & 29 & 1.9945 & .84608 & .15711 & 1.6727 & 2.3163 & .00 & 4.13 & 3.69 & 0.30 \\
\hline C67 & 29 & 2.1941 & .97373 & .18082 & 1.8238 & 2.5645 & .45 & 3.87 & 4.14 & 0.25 \\
\hline Average & 145 & 2.2117 & .79891 & .06635 & 2.0805 & 2.3428 & .00 & 4.45 & 3.81 & 0.61 \\
\hline Left anteric & facet & sagittal) & & & & & & & & \\
\hline C23 & 29 & .7966 & .30543 & .05672 & .6804 & .9127 & .00 & 1.32 & 1.41 & 0.19 \\
\hline C34 & 29 & .8421 & .29392 & .05458 & .7303 & .9539 & .24 & 1.43 & 1.43 & 0.25 \\
\hline C45 & 29 & .9179 & .26513 & .04923 & .8171 & 1.0188 & .38 & 1.44 & 1.45 & 0.39 \\
\hline C56 & 29 & .9355 & .39853 & .07401 & .7839 & 1.0871 & .00 & 1.76 & 1.73 & 0.14 \\
\hline C67 & 29 & .9038 & .39755 & .07382 & .7526 & 1.0550 & .00 & 1.65 & 1.70 & 0.11 \\
\hline Average & 145 & .8792 & .33606 & .02791 & .8240 & .9343 & .00 & 1.76 & 1.55 & 0.21 \\
\hline Right anter & or fac & (sagittal & & & & & & & & \\
\hline $\mathrm{C} 23$ & 29 & .8566 & .38400 & .07131 & .7105 & 1.0026 & .21 & 1.83 & 1.62 & 0.09 \\
\hline C34 & 29 & .8648 & .27684 & .05141 & .7595 & .9701 & .33 & 1.36 & 1.42 & 0.31 \\
\hline C45 & 29 & .9162 & .33646 & .06248 & .7882 & 1.0442 & .00 & 1.62 & 1.59 & 0.24 \\
\hline C56 & 29 & .8821 & .37869 & .07032 & .7380 & 1.0261 & .00 & 1.52 & 1.64 & 0.12 \\
\hline C67 & 29 & .7931 & .30833 & .05725 & .6758 & .9104 & .00 & 1.54 & 1.41 & 0.18 \\
\hline Average & 145 & .8626 & .33704 & .02799 & .8072 & .9179 & .00 & 1.83 & 1.54 & 0.19 \\
\hline Left posteri & r face & (sagittal) & & & & & & & & \\
\hline $\mathrm{C} 23$ & 29 & .6290 & .29975 & .05566 & .5149 & .7430 & .00 & 1.22 & 1.23 & 0.03 \\
\hline C34 & 29 & .7166 & .29220 & .05426 & .6054 & .8277 & .00 & 1.23 & 1.30 & 0.13 \\
\hline $\mathrm{C} 45$ & 29 & .6634 & .27198 & .05051 & .5600 & .7669 & .14 & 1.18 & 1.21 & 0.12 \\
\hline C56 & 29 & .7324 & .29715 & .05518 & .6194 & .8454 & .26 & 1.51 & 1.33 & 0.14 \\
\hline C67 & 29 & .5210 & .26208 & .04867 & .4213 & .6207 & .21 & 1.29 & 1.05 & 0.00 \\
\hline Average & 145 & .6525 & .29105 & .02417 & .6047 & .7003 & .00 & 1.51 & 1.23 & 0.07 \\
\hline Right poste & ior $\mathrm{fa}$ & t (sagitta & & & & & & & & \\
\hline $\mathrm{C} 23$ & 29 & .6834 & .25688 & .04770 & .5857 & .7812 & .13 & 1.21 & 1.20 & 0.17 \\
\hline C34 & 29 & .7752 & .37371 & .06940 & .6330 & .9173 & .00 & 1.39 & 1.52 & 0.03 \\
\hline $\mathrm{C} 45$ & 29 & .6862 & .30326 & .05631 & .5709 & .8016 & .24 & 1.53 & 1.29 & 0.08 \\
\hline C56 & 29 & .7872 & .29044 & .05393 & .6768 & .8977 & .31 & 1.37 & 1.37 & 0.21 \\
\hline C67 & 29 & .6003 & .25693 & .04771 & .5026 & .6981 & .00 & 1.12 & 1.11 & 0.09 \\
\hline Average & 145 & .7065 & .30303 & .02517 & .6567 & .7562 & .00 & 1.53 & 1.31 & 0.10 \\
\hline Left middle & facet & gittal) & & & & & & & & \\
\hline C23 & 29 & .9766 & .39113 & .07263 & .8278 & 1.1253 & .00 & 2.15 & 1.76 & 0.19 \\
\hline C34 & 29 & 1.2566 & .39229 & .07285 & 1.1073 & 1.4058 & .60 & 2.04 & 2.04 & 0.47 \\
\hline C45 & 29 & 1.2341 & .39665 & .07366 & 1.0833 & 1.3850 & .57 & 2.21 & 2.03 & 0.44 \\
\hline C56 & 29 & 1.1710 & .32102 & .05961 & 1.0489 & 1.2931 & .48 & 1.74 & 1.81 & 0.53 \\
\hline C67 & 29 & .9700 & .32733 & .06078 & .8455 & 1.0945 & .43 & 1.58 & 1.62 & 0.32 \\
\hline Average & 145 & 1.1217 & .38303 & .03181 & 1.0588 & 1.1845 & .00 & 2.21 & 1.89 & 0.36 \\
\hline Right midd & facet & sagittal) & & & & & & & & \\
\hline C23 & 29 & .9917 & .35350 & .06564 & .8573 & 1.1262 & .46 & 1.84 & 1.70 & 0.28 \\
\hline C34 & 29 & 1.2634 & .50039 & .09292 & 1.0731 & 1.4538 & .45 & 2.45 & 2.26 & 0.26 \\
\hline C45 & 29 & 1.3114 & .48074 & .08927 & 1.1285 & 1.4942 & .20 & 2.39 & 2.27 & 0.35 \\
\hline C56 & 29 & 1.3210 & .51341 & .09534 & 1.1257 & 1.5163 & .37 & 2.41 & 2.35 & 0.29 \\
\hline C67 & 29 & .9717 & .37353 & .06936 & .8296 & 1.1138 & .41 & 2.11 & 1.72 & 0.22 \\
\hline Average & 145 & 1.1719 & .47011 & .03904 & 1.0947 & 1.2490 & .20 & 2.45 & 2.11 & 0.23 \\
\hline
\end{tabular}


Table 2. Descriptive statistics of cervical measurements with sides combined.

\begin{tabular}{|c|c|c|c|c|c|c|c|c|c|}
\hline & \multirow[b]{2}{*}{ Mean } & \multirow[b]{2}{*}{ SD } & \multirow{2}{*}{$\begin{array}{c}\text { Standard } \\
\text { Error }\end{array}$} & \multicolumn{2}{|c|}{ 95\% CI for Mean } & \multirow[b]{2}{*}{ Minimum } & \multirow[b]{2}{*}{ Maximum } & \multirow[b]{2}{*}{ 95\% Upper CI } & \multirow[b]{2}{*}{ 95\% Lower CI } \\
\hline & & & & Lower Bound & Upper Bound & & & & \\
\hline UVJ (coror & & & & & & & & & \\
\hline $\mathrm{C} 23$ & 1.75 & .827 & .154 & 1.43 & 2.06 & 1 & 4 & 3.40 & 0.09 \\
\hline C34 & 1.71 & .794 & .148 & 1.41 & 2.01 & 1 & 4 & 3.30 & 0.12 \\
\hline $\mathrm{C} 45$ & 1.41 & .610 & .114 & 1.17 & 1.64 & 0 & 3 & 2.63 & 0.19 \\
\hline C56 & 1.27 & .565 & .105 & 1.06 & 1.49 & 0 & 3 & 2.40 & 0.14 \\
\hline C67 & 1.87 & .714 & .133 & 1.60 & 2.14 & 1 & 4 & 3.30 & 0.44 \\
\hline Average & 1.60 & .737 & .061 & 1.48 & 1.72 & 0 & 4 & 3.08 & 0.13 \\
\hline Middle disc & nid coron & & & & & & & & \\
\hline $\mathrm{C} 23$ & 4.9972 & .73483 & .13645 & 4.7177 & 5.2768 & 3.25 & 6.19 & 6.47 & 3.53 \\
\hline C34 & 4.7810 & 1.04291 & .19366 & 4.3843 & 5.1777 & 2.22 & 7.10 & 6.87 & 2.70 \\
\hline $\mathrm{C} 45$ & 4.8176 & 1.15149 & .21383 & 4.3796 & 5.2556 & 1.12 & 6.48 & 7.12 & 2.51 \\
\hline C56 & 4.6193 & 1.09779 & .20386 & 4.2017 & 5.0369 & 1.93 & 6.62 & 6.81 & 2.42 \\
\hline C67 & 5.0262 & .95944 & .17816 & 4.6613 & 5.3912 & 2.52 & 6.95 & 6.95 & 3.11 \\
\hline Average & 4.8483 & 1.00505 & .08347 & 4.6833 & 5.0133 & 1.12 & 7.10 & 6.86 & 2.84 \\
\hline Medial face & coronal) & & & & & & & & \\
\hline C23 & .5881 & .23681 & .04397 & .4980 & .6782 & .13 & 1.08 & 1.06 & 0.11 \\
\hline C34 & .6033 & .22338 & .04148 & .5183 & .6882 & .17 & 1.04 & 1.05 & 0.16 \\
\hline $\mathrm{C} 45$ & .5438 & .26429 & .04908 & .4433 & .6443 & .16 & 1.21 & 1.07 & 0.02 \\
\hline C56 & .6840 & .31875 & .05919 & .5627 & .8052 & .00 & 1.54 & 1.32 & 0.05 \\
\hline C67 & .4993 & .22690 & .04213 & .4130 & .5856 & .10 & .96 & 0.95 & 0.05 \\
\hline Average & .5837 & .26098 & .02167 & .5409 & .6265 & .00 & 1.54 & 1.11 & 0.06 \\
\hline Midpoint $\mathrm{f}$ & t (coronal & & & & & & & & \\
\hline C23 & 1.05 & .400 & .074 & .90 & 1.20 & 0 & 2 & 1.85 & 0.25 \\
\hline C34 & 1.33 & .448 & .083 & 1.16 & 1.50 & 0 & 2 & 2.23 & 0.44 \\
\hline $\mathrm{C} 45$ & 1.37 & .424 & .079 & 1.21 & 1.53 & 1 & 2 & 2.22 & 0.52 \\
\hline C56 & 1.26 & .443 & .082 & 1.09 & 1.43 & 0 & 3 & 2.15 & 0.38 \\
\hline C67 & 1.04 & .386 & .072 & .89 & 1.19 & 0 & 2 & 1.81 & 0.27 \\
\hline Average & 1.21 & .441 & .037 & 1.14 & 1.28 & 0 & 3 & 2.09 & 0.33 \\
\hline Lateral face & (coronal) & & & & & & & & \\
\hline $\mathrm{C} 23$ & .4995 & .23578 & .04378 & .4098 & .5892 & .00 & 1.10 & 0.97 & 0.03 \\
\hline C34 & .4807 & .22524 & .04183 & .3950 & .5664 & .12 & .99 & 0.93 & 0.03 \\
\hline $\mathrm{C} 45$ & .5438 & .25153 & .04671 & .4481 & .6395 & .12 & 1.12 & 1.05 & 0.04 \\
\hline C56 & .5179 & .28736 & .05336 & .4086 & .6272 & .00 & 1.41 & 1.09 & 0.00 \\
\hline C67 & .5159 & .23610 & .04384 & .4261 & .6057 & .00 & 1.07 & 0.99 & 0.04 \\
\hline Average & .5116 & .24795 & .02059 & .4709 & .5523 & .00 & 1.41 & 1.01 & 0.02 \\
\hline ISD (sagitt & & & & & & & & & \\
\hline $\mathrm{C} 23$ & 15.7624 & 2.75350 & .51131 & 14.7150 & 16.8098 & 7.70 & 20.95 & 21.27 & 10.26 \\
\hline C34 & 14.6317 & 1.76001 & .32683 & 13.9623 & 15.3012 & 12.24 & 19.16 & 18.15 & 11.11 \\
\hline $\mathrm{C} 45$ & 14.0155 & 1.84557 & .34271 & 13.3135 & 14.7175 & 10.09 & 16.52 & 17.71 & 10.32 \\
\hline C56 & 13.1521 & 1.56348 & .29033 & 12.5574 & 13.7468 & 10.32 & 16.47 & 16.28 & 10.03 \\
\hline C67 & 14.1241 & 1.81533 & .33710 & 13.4336 & 14.8147 & 11.46 & 19.20 & 17.75 & 10.49 \\
\hline Average & 14.3372 & 2.14344 & .17800 & 13.9853 & 14.6890 & 7.70 & 20.95 & 18.62 & 10.05 \\
\hline Vertebral b & y translat & n (sagittal) & & & & & & & \\
\hline C23 & .5841 & .86261 & .16018 & .2560 & .9123 & -1.09 & 3.86 & 2.31 & 0.00 \\
\hline C34 & .5369 & .64093 & .11902 & .2931 & .7807 & -.84 & 1.74 & 1.82 & 0.00 \\
\hline $\mathrm{C} 45$ & .5210 & .72608 & .13483 & .2448 & .7972 & -1.57 & 1.70 & 1.97 & 0.00 \\
\hline C56 & .0855 & .88789 & .16488 & -.2522 & .4233 & -1.53 & 1.93 & 1.86 & 0.00 \\
\hline C67 & .2131 & .91067 & .16911 & -.1333 & .5595 & -1.50 & 1.45 & 2.03 & 0.00 \\
\hline Average & .3881 & .82582 & .06858 & .2526 & .5237 & -1.57 & 3.86 & 2.04 & 0.00 \\
\hline Anterior D & (sagittal) & & & & & & & & \\
\hline C23 & 2.6990 & .83906 & .15581 & 2.3798 & 3.0181 & 1.13 & 4.40 & 4.38 & 1.02 \\
\hline C34 & 2.8310 & .85043 & .15792 & 2.5075 & 3.1545 & 1.05 & 4.32 & 4.53 & 1.13 \\
\hline $\mathrm{C} 45$ & 3.4552 & .94400 & .17530 & 3.0961 & 3.8143 & 1.42 & 5.89 & 5.34 & 1.57 \\
\hline C56 & 3.1310 & 1.15688 & .21483 & 2.6910 & 3.5711 & .00 & 5.19 & 5.44 & 0.82 \\
\hline C67 & 3.4262 & 1.10487 & .20517 & 3.0059 & 3.8465 & .99 & 5.26 & 5.64 & 1.22 \\
\hline Average & 3.1085 & 1.02094 & .08478 & 2.9409 & 3.2761 & .00 & 5.89 & 5.15 & 1.07 \\
\hline Middle DH & agittal) & & & & & & & & \\
\hline C23 & 4.888 & .7602 & .1412 & 4.598 & 5.177 & 3.0 & 6.4 & 6.41 & 3.37 \\
\hline C34 & 4.848 & 1.1929 & .2215 & 4.394 & 5.301 & 1.8 & 7.1 & 7.23 & 2.46 \\
\hline $\mathrm{C} 45$ & 4.922 & 1.1833 & .2197 & 4.472 & 5.372 & 1.1 & 6.7 & 7.29 & 2.56 \\
\hline C56 & 4.656 & 1.2052 & .2238 & 4.198 & 5.115 & 1.8 & 6.5 & 7.07 & 2.25 \\
\hline C67 & 5.244 & 1.1833 & .2197 & 4.794 & 5.694 & 1.3 & 6.7 & 7.61 & 2.88 \\
\hline Average & 4.911 & 1.1191 & .0929 & 4.728 & 5.095 & 1.1 & 7.1 & 7.15 & 2.67 \\
\hline Posterior D & (sagittal) & & & & & & & & \\
\hline C23 & 2.3107 & .70818 & .13151 & 2.0413 & 2.5801 & .99 & 4.45 & 3.73 & 0.89 \\
\hline C34 & 2.1069 & .74378 & .13812 & 1.8240 & 2.3898 & .43 & 3.56 & 3.59 & 0.62 \\
\hline $\mathrm{C} 45$ & 2.4521 & .65815 & .12222 & 2.2017 & 2.7024 & 1.07 & 3.74 & 3.77 & 1.14 \\
\hline C56 & 1.9945 & .84608 & .15711 & 1.6727 & 2.3163 & .00 & 4.13 & 3.69 & 0.30 \\
\hline C67 & 2.1941 & .97373 & .18082 & 1.8238 & 2.5645 & .45 & 3.87 & 4.14 & 0.25 \\
\hline Average & 2.2117 & .79891 & .06635 & 2.0805 & 2.3428 & .00 & 4.45 & 3.81 & 0.61 \\
\hline
\end{tabular}


Table 2. Continued.

\begin{tabular}{|c|c|c|c|c|c|c|c|c|c|}
\hline & \multirow[b]{2}{*}{ Mean } & \multirow[b]{2}{*}{ SD } & \multirow{2}{*}{$\begin{array}{c}\text { Standard } \\
\text { Error }\end{array}$} & \multicolumn{2}{|c|}{ 95\% CI for Mean } & \multirow[b]{2}{*}{ Minimum } & \multirow[b]{2}{*}{ Maximum } & \multirow[b]{2}{*}{ 95\% Upper CI } & \multirow[b]{2}{*}{ 95\% Lower CI } \\
\hline & & & & Lower Bound & Upper Bound & & & & \\
\hline \multicolumn{10}{|c|}{ Anterior facet (sagittal) } \\
\hline C23 & .8266 & .34472 & .06401 & .6954 & .9577 & .00 & 1.83 & 1.52 & 0.14 \\
\hline C34 & .8534 & .28538 & .05299 & .7449 & .9620 & .24 & 1.43 & 1.42 & 0.28 \\
\hline C45 & .9171 & .30079 & .05586 & .8027 & 1.0315 & .00 & 1.62 & 1.52 & 0.32 \\
\hline C56 & .9088 & .38861 & .07216 & .7610 & 1.0566 & .00 & 1.76 & 1.69 & 0.13 \\
\hline C67 & .8484 & .35294 & .06554 & .7142 & .9827 & .00 & 1.65 & 1.55 & 0.14 \\
\hline Average & .8709 & .33655 & .02795 & .8156 & .9261 & .00 & 1.83 & 1.54 & 0.20 \\
\hline \multicolumn{10}{|c|}{ Posterior facet (sagittal) } \\
\hline $\mathrm{C} 23$ & .6562 & .27832 & .05168 & .5503 & .7621 & .00 & 1.22 & 1.21 & 0.10 \\
\hline C34 & .7459 & .33296 & .06183 & .6192 & .8725 & .00 & 1.39 & 1.41 & 0.08 \\
\hline $\mathrm{C} 45$ & .6748 & .28762 & .05341 & .5654 & .7842 & .14 & 1.53 & 1.25 & 0.10 \\
\hline C56 & .7598 & .29380 & .05456 & .6481 & .8716 & .26 & 1.51 & 1.35 & 0.17 \\
\hline C67 & .5607 & .25950 & .04819 & .4620 & .6594 & .00 & 1.29 & 1.08 & 0.04 \\
\hline Average & .6795 & .29704 & .02467 & .6307 & .7282 & .00 & 1.53 & 1.27 & 0.09 \\
\hline \multicolumn{10}{|c|}{ Middle facet (sagittal) } \\
\hline C23 & .9841 & .37232 & .06914 & .8425 & 1.1258 & .00 & 2.15 & 1.73 & 0.24 \\
\hline C34 & 1.2600 & .44634 & .08288 & 1.0902 & 1.4298 & .45 & 2.45 & 2.15 & 0.37 \\
\hline C45 & 1.2728 & .43870 & .08146 & 1.1059 & 1.4396 & .20 & 2.39 & 2.15 & 0.40 \\
\hline C56 & 1.2460 & .41721 & .07747 & 1.0873 & 1.4047 & .37 & 2.41 & 2.08 & 0.41 \\
\hline C67 & .9709 & .35043 & .06507 & .8376 & 1.1042 & .41 & 2.11 & 1.67 & 0.27 \\
\hline Average & 1.1468 & .42657 & .03542 & 1.0767 & 1.2168 & .00 & 2.45 & 2.00 & 0.29 \\
\hline
\end{tabular}

bony, CT based criteria would obviate the need for MRI to screen for occult trauma in some cases.

Our results also indicate that vertebral body translation should be less than $2 \mathrm{~mm}$ in the anteriorposterior direction of supine CT scan. In contrast, the previously reported guideline was less than 3.5 $\mathrm{mm}$ of translation is physiologic based upon early biomechanical studies. Previous radiographic studies have referenced the posterior vertebral body and spinolaminar lines. We suspect that absence of translation of subaxial vertebral bodies would result in the linear orientation, which would be visualized on radiographs. We believe that the CT based measurement technique is superior to conventional radiography because our normative value is an order of magnitude less than the current reference standard. Additionally, existing radiographic landmarks, such as the spinolaminar line, may be impractical on $\mathrm{CT}$ if the spinous processes and laminae in the same plane due to rotation of the head or neck. The translation upper limit of $2 \mathrm{~mm}$ is also small and can be measured in either the anterior or posterior direction. In contrast, the upper limit of the ISD was $18.6 \mathrm{~mm}$.

Although interspinous widening and $\mathrm{DH}$ are considered to be important markers of posterior

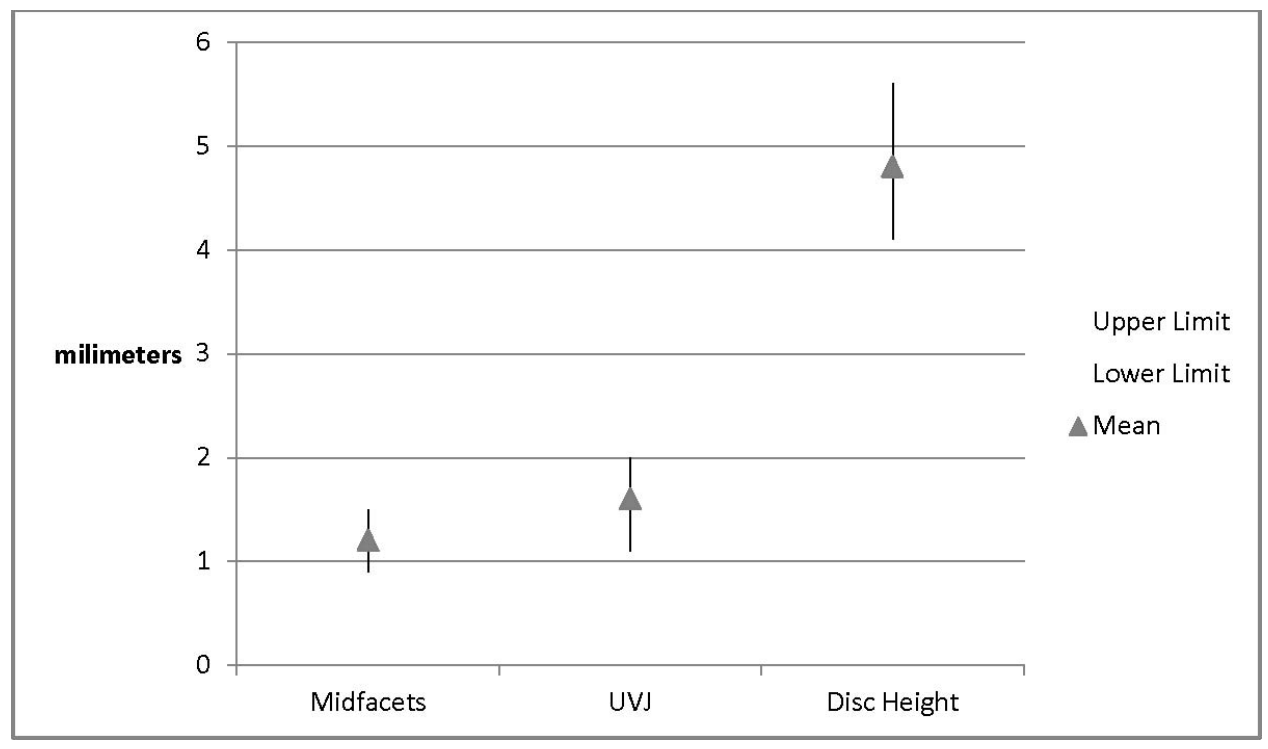

Figure 3. Combined right and left facet and UVJ 95\% Cls and DH 95\% Cl. 
ligamentous injury, DH and ISD are unlikely to be as useful as screening measurements because of wider variation in CIs. We believe that the measurements of $\mathrm{DH}$ and uncovertebral height may be susceptible to spondylosis. The upper limit of normal DH was $5.2 \mathrm{~mm}$ anteriorly, $7.15 \mathrm{~mm}$ in the midpoint, and $3.81 \mathrm{~mm}$ posteriorly. Not surprisingly, both the facet and the UVJ measurements were small. The facet joints were superior to UVJs as a measurement landmark because we did not identify as much variation between anatomical levels in the facet joint measurements as we observed in the UVJ measurements.

There are concerns or potential limitations in the methodology. While this software has been used for multiple published studies and has the ability to measure to the nearest 100th of a millimeter, the actual interrater and intrarater reliability have never been tested. All measurements were made by the primary author who had extensive experience with this software. The apparent repeatability of the data measurements along with the narrow CIs suggest very little variation in measurement. While the final sample size appeared small, CIs were narrow, SDs low, and power analysis supported an adequate number of measurements. The sample population could generate selection bias as these subjects were all involved in some sort of trauma, but in turn likely represent valid normative data for trauma patients for which this study was intended. These patients were all radiographically and clinically normal with short-term follow-up. Although it would be interesting to validate these measurements as a screening tool in a population of patients with pathology, unfortunately, this population was derived from a normative database only. We do not have information on patients with pathology such as facet dislocation. Future studies will explore this topic. Extrapolating to the population at large may be a limitation. In particular, there may be some populations (e.g., adolescents, elderly patients) who have slightly different anatomical measurements. Nevertheless, the threshold values chosen in this study represent the $95 \%$ upper limit of normal. The main limitation is probably practical application. The software used for measurements enabled additional functionality such as reorientation of the planes of measurement and interactive zoom, which may not be available on all commercially available software programs. While this provided accurate and reproducible data, this may limit the clinical applicability of this technique. In addition, though most clinicians are aware, it should be stated that these numbers will change with out of plane imaging. As always, diagnostic interpretation is more difficult and less accurate with suboptimal imaging. This supports quality assurance and performance improvement efforts to optimize the methodology of image acquisition and reconstruction. In addition, radiological evaluation software continues to improve every year with improving capacity to reorient reconstructive imaging in proper planes. With time, this will likely be standard, making these data even more valuable. Our data were also not normally distributed, but the CI calculation (of mean plus or minus two SDs) assumes a normal distribution. However, this criticism applies to many of the statistical calculations (including mean and SD). Ranges were also reported in the accompanying tables. Finally, these measurements represent bony displacement. It is possible that a patient could have an unstable injury but have very low bony displacement measurements due to positioning during the CT scan.

It is also important to interpret results in the context of overall clinical finding. There can be baseline degenerative, inflammatory, or congenital anomalies that are stable, chronic relationships, but contribute to alignment variability.

In summary, normal cervical spine quantitative relationships have been described. From these data, screening measurements to suggest potential abnormal alignment were identified. Facet and intervertebral translation measurements seem to have the most value in this regard with upper limits of 1.5 $\mathrm{mm}$ and $2 \mathrm{~mm}$, respectively. DH and ISDs are more variable and thus likely less sensitive, and demonstrated upper limits of $7 \mathrm{~mm}$ and $18.6 \mathrm{~mm}$, respectively. Future study to compare a cohort of subaxial cervical spine patients with traumatic injuries and in particular missed injuries would help validate the value of these data.

\section{REFERENCES}

1. Como JJ, Diaz JJ, Dunham CM, et al. Practice management guidelines for identification of cervical spine injuries following trauma: update from the eastern association for the surgery of trauma practice management guidelines committee. J Trauma. 2009;67(3):651-659.

2. Berne JD, Velmahos GC, El-Tawil Q, et al. Value of complete cervical helical computed tomographic scanning in identifying cervical spine injury in the unevaluable blunt trauma 
patient with multiple injuries: a prospective study. J Trauma. 1999;47(5):896-902; discussion 902-903.

3. Brohi K, Healy M, Fotheringham T, et al. Helical computed tomographic scanning for the evaluation of the cervical spine in the unconscious, intubated trauma patient. $J$ Trauma. 2005;58(5):897-901.

4. Diaz JJ Jr, Gillman C, Morris JA, et al. Are five-view plain films of the cervical spine unreliable? A prospective evaluation in blunt trauma patients with altered mental status. $J$ Trauma. 2003;55(4):658-663; discussion 663-664.

5. Griffen MM, Frykberg ER, Derwin AJ, et al. Radiographic clearance of blunt cervical spine injury: plain radiograph or computed tomography scan? J Trauma. 2003;55(2):222-226; discussion 226-227.

6. Holmes JF, Akkinepalli R. Computed tomography versus plain radiography to screen for cervical spine injury: a metaanalysis. J Trauma. 2005;58(5):902-905.

7. Mathen R, Inaba K, Munera F, et al. Prospective evaluation of multislice computed tomography versus plain radiographic cervical spine clearance in trauma patients. $J$ Trauma. 2007;62:1427-1431.

8. Tomycz ND, Chew BG, Chang YF, et al. MRI is unnecessary to clear the cervical spine in obtunded/comatose trauma patients: the four-year experience of a level I trauma center. J Trauma. 2008;64(5):1258-1263.

9. Radcliff KE, Ben-Galim P, Dreiangel N, et al. Comprehensive computed tomography assessment of the upper cervical anatomy: what is normal? Spine J. 2010;10(3):219-229.
10. White AA, Johnson RM, Panjabi MM, et al. Biomechanical analysis of clinical stability in the cervical spine. Clin Orthopaedics Related Res. 1975(109):85-96.

11. Bono CM, Vaccaro AR, Fehlings M, et al. Measurement techniques for upper cervical spine injuries: consensus statement of the Spine Trauma Study Group. Spine. 2007;32(5):593-600.

Disclosures and COI: There was no funding source for this manuscript. This was approved by the Office of Research Institutional Review Board, Baylor College of Medicine.

Corresponding Author: Charles A. Reitman, MD, MUSC Department of Orthopaedics, 96 Jonathan Lucas St, 708 CSB, Charleston, SC 29425. Phone: (843)-792-8959; Fax: (843)-7923674; Email: reitman@musc.edu.

Published 31 August 2018

This manuscript is generously published free of charge by ISASS, the International Society for the Advancement of Spine Surgery. Copyright (c) 2018 ISASS. To see more or order reprints or permissions, see http://ijssurgery.com. 